\title{
HoNOS v. GP opinion in a shifted out-patient clinic
}

\author{
John R. Taylor and Greg Wilkinson
}

\begin{abstract}
This study compared the mean change in the total Heatth of the Nation Outcome Scales (HONOS) with the general practtioners' (GP3) assessment of clinical improvement and satisfaction, in a shiffed peychiatric out-patient clinic. The total HoNOS fell significantly between the first and last appointment, suggesting that it was sensitive to change even in patients with a lower level of morbidity. There was a weak association between change in the total HoNOS and the GPs' assesement of clinical improvement $(r=0.4 ; P=0.06)$, and a trend towards a greater HoNOS fall in patients that the GPs assessed as having improved clinically (5.3) compared with those the GP thought had not changed (2.5), but this difference was not significant (95\% Cl, 6.6 to-1.1). There was no evidence of a relationship between change in HoNOS and GP satisfaction, and no trend towards a greater fall in the HoNOS in cases where the GP was satistied, desplte a lack of improvement being the commonest reason olven by GPs for allseatisfaction. The results suggest that the foll in total HoNOS relates to some degree with GPs' own aseosement of clinical improvement in their patients, and that the HoNOS may be regarded as useful by GP functholders.
\end{abstract}

One of the three mental health targets set out in the Health of the Nation (Department of Health, 1992) was to improve the health and social functioning of mentally ill people. Health of the Nation Outcome Scales (HoNOS) were developed as a simple way of measuring mental health and social functioning that would be reliable, sensitive to change, and cover both clinical and social problems. It was designed to be used routinely in any setting, but the initial field trials chiefly involved patients with severe mental lllness (Wing et al, 1996). The planning and priorities guidance for 1997/8 (NHS Executive, 1996) encourages health authorities to establish a basis for the introduction of the HoNOS.

Previous evaluation of the HoNOS has not involved any assessment of whether it will be useful to commissioning services. Unless the HoNOS agrees with a general practitioner's (GP) own assessment of improvement in their patients, it is unlikely that GP fundholders will regard the HoNOS as useful.
This study compared the fall in the total HoNOS at the first and last appointment with the GP's assessment or improvement in their patient's symptoms of functioning and with their overall satisfaction.

\section{The study}

All referrals from GPs to a shifted out-patient clinic in a primary care health centre over a tenmonth period were included in the study. The clinic was located in Speke which is a geographically distinct area on the outskirts of Liverpool. It has a high underprivileged area score of 37.92 and a population of around 12000 . Primary care services were provided by eight GPs based at three separate sites. Only one of these GPs was a fundholder. HoNOS were completed on all patients at their first and last appointment by the same psychiatrist (J. T.). Differences in the HoNOS were compared using a paired $t$-test. The results were not analysed by separate scale items due to the small sample size.

GPs were interviewed at the end of the study period and asked if they thought the patients' symptoms and functioning had improved since referral and how satisfied they were, overall, with each referral. Particular attention was given to the attribution of any mixed satisfaction to provide information on why, not just whether, GPs were satisfled and overcome some of the limitations of satisfaction surveys (Williams \& Wilkinson, 1995). No GP thought that their patients had deteriorated or expressed only negative satisfaction. Pearson correlation coefficients and unpaired $t$-tests were used to compare the change in the HoNOS with the opinion of the GP.

\section{Findings}

Patients' characteristics

There were 47 referrals during the study period and $81 \%(38 / 47)$ attended at least one outpatient appointment. The mean age of the 
referrals was 36.4 years (s.d. 11.1 , range 18 to $59)$ and $49 \%(23 / 47)$ were male. Half of the outpatients $(19 / 38)$ seen had a clinical diagnosis of depression (ICD-10 F32-33.2); a quarter had neurotic or anxiety disorders (F40-42); $15 \%$ had stress-related or eating disorders (F43, F50); two had schizophrenia (F20); and one had a personality disorder (F60). Less than a third had been previously seen by a psychiatrist (12/ 38).

\section{Health of the Nation Outcome Scale}

The average total HoNOS score on the first appointment for the 39 patients who attended was 10.7 (s.d. 3.4, range 4 to 23). Twenty-six patients were seen two or more times at the outpatient clinic. The mean total fell significantly from 11.1 (s.d. 3.5; range 5 to 23 ) on the first appointment to 6.8 (s.d. 3.1; range 0 to 14 ) at the last $(t=5.1 ; 95 \% C I, 2.5$ to 6.0$)$. The GPs had seen 22 of these patients again, and reported that their symptoms had improved in 57\% (12/22). There was a trend (see Table 1) for a greater mean fall in the HoNOS for the patients that the GPs felt had improved (5.3), compared with patients that the GPs felt had not improved (2.5), but this difference was not significant (95\% CI, 6.6 to -1.1$)$. The correlation coefficient was $0.41 \quad(P=0.06)$, suggesting that there was a weak relationship between change in total HoNOS and GPs' assessment of clinical improvement.

\section{HoNOS and satisfaction with referral}

GPs were at least mostly satisfied with $68 \%(15)$ 22) of referrals seen two or more times. This level of satisfaction was similar to that for all patients seen again by the GP after referral $(26 / 38)$. The HoNOS fell an average of 4.26 in referrals where the GP was at least mostly satisfied, compared with 3.50 when the GP reported mixed satisfaction (see Table 2). This difference was not significant $(95 \% \mathrm{CI}, 5.4$ to -3.2$)$ and the correlation coefficient was $0.08 \quad(P=0.69)$. This suggests that there is no association between GP satisfaction and change in the HoNOS, despite lack of improvement in the patient being the commonest attribution given by GPs for mixed satisfaction.

\section{Comment}

The total HoNOS fell significantly between the first and last appointment, suggesting that it is sensitive to change even in patients with lower morbidity attending a shifted out-patient clinic. There was evidence of a weak relationship between the GPs' assessment of clinical improvement and change in the total HoNOS. Although this relationship was not significant, the results suggest that HoNOS may be regarded by GP fundholders as a relevant and useful outcome measure. The comparative lack of any association between GP satisfaction and the fall in the HoNOS is puzzling, as the main reason given for dissatisfaction was lack of change in the patient. It is known that there is often no clear clinical indication for GP referrals (Kaeser \& Cooper, 1971) and that GPs often refer to reduce stress and calls on their time (Robertson, 1979). It is possible that the GPs' satisfaction is partly related to non-clinical factors, such as the burden they feel under, and this explains the lack of association with HoNOS, which measures outcome in the patient.

Only limited conclusions can be drawn from this study due to its small size and because the GPs and psychiatrist were rating the patients at different points in time. Information on the GPs'

Table 1. Mean change in HoNOS compared with GPs' estimated improvement in symptoms

\begin{tabular}{lll}
\hline GP' eettimate of outcome & Mean change in HoNOS & No. patients \\
\hline Improved a lot & 6.7 & 7 \\
Improved a little & 3.2 & 5 \\
No change & 2.5 & 10 \\
Total & 4.0 & 22 \\
\hline
\end{tabular}

Table 2. Mean change in HoNOS compared with GPs' satisfaction

\begin{tabular}{llc}
\hline GPs' satisfaction & Mean change in HoNOS & No. patients \\
\hline Excellent & 4.6 & 5 \\
Mostly satisfied & 4.0 & 11 \\
Mixed & 3.5 & 6 \\
Total & 4.0 & 22 \\
\hline
\end{tabular}


opinions of the patients' progress may also be biased due to the number of patients who dropped out of follow-up. These same limitations are likely to apply if the HoNOS are used to provide information to GP fundholders, and this study suggests that the HoNOS will be seen by GPs as a useful measure of outcome. The routine use of HoNOS may have the additional benefit of focusing service developments on those that improve the health or social functioning of patients rather than those developments that satisfy purchasers.

\section{References}

DEPARTMENT OF HEALTH (1992) The Health of the Nation: A Strategy of Health for England. London: HMSO.

KAESER, A. C. \& COOPER, B. (1971) The psychiatric patient. the general practitioner, and the outpatient clinic: an operational study and review. Psychological Medicine, 1. 312-325.
NHS EXECUTIVE (1996) Planning and Prlortties Guidance for the NHS 1997/8. Leeds: Department of Health.

ROBERTSON. N. C. (1979) Variations in referral pattern to the psychiatric services by general practitioners. Psychological Medictine, 8. 355-364.

WiLuAMS, B. \& Wilkinson, G. (1995) Patient satisfaction in mental care: evaluating an evaluative method. Brtish Journal of Psychiatry. 168, 559-562.

WING. J. K., CURTIS, R. H. \& BEEVOR. A. S. (1996) HoNOS: Health of the Nation Outcome Scales. Report on Research and Development. London: Royal College of Psychiatrists Research Unit.

* John R. Taylor, Senior Registrar, North Mersey Community Trust, Mossley Hill Hospital, Park Avenue, Liverpool L18 8BU; and Greg Wilkinson, Professor of Liaison Psychiatry. Department of Psychiatry, Royal Liverpool University Hospital, Liverpool L69 3BX

*Correspondence 